\title{
CAMBIOS EN LA DISTRIBUCIÓN GEOGRÁFICA DE ALGUNAS ESPECIES AMERICANAS EN LA PROVINCIA DE BUENOS AIRES
}

\author{
Vercelli, N. ${ }^{1,2,3 ;}$ Scaramuzzino, R. L. ${ }^{1 ;}$ D’Alfonso, C. ${ }^{1}$ \\ MILIONE, G. ${ }^{1} \&$ PIAZZA, G. ${ }^{1}$
}

\begin{abstract}
RESUMEN
La intensificación de las actividades antrópicas produce cambios en los paisajes que pueden producir modificaciones en la distribución de las especies. En la provincia de Buenos Aires se registraron plantas y animales que extendieron su hábitat hacia el sur. Aquí se analizan los casos de Glinus radiatus, Guilleminea densa y Thalia multiflora, halladas actualmente a más de 250 km del límite sur de su localización conocida. Se recolectaron ejemplares en los partidos de Azul, Las Flores y Rojas, y se revisó la bibliografía existente para ubicar registros previos y discutir las causas de su expansión. Entre las hipótesis que explican el avance de estas especies se destaca los cambios registrados en las precipitaciones y temperaturas de la región. También es posible que la restringida localización previamente publicada se deba a la insuficiencia de relevamiento, o bien los ejemplares hallados podrían constituir poblaciones relictuales, o haberse expandido favorecidos por corredores antrópicos.
\end{abstract}

Key words: Glinus radiatus, Guilleminea densa, Thalia multiflora, distribución geográfica, provincia de Buenos Aires.

1.- Facultad de Agronomía, Universidad Nacional del Centro de la Provincia de Buenos Aires (UNCPBA) República de Italia 780 (B7300). Azul, Buenos Aires, Argentina. E-mail: nvercelli@ihlla.org.ar

2.- Consejo Nacional de Investigaciones Científicas y Técnicas (CONICET). Godoy Cruz 2290 (C1425FQB) CABA, Argentina.

3.- Instituto de Hidrología de Llanuras “Dr. Eduardo J. Usunoff” (UNCPBA - CIC - CONICET). República de Italia 780. (B7300) Azul, Buenos Aires, Argentina.

Manuscrito recibido el 11 de abril de 2020 y aceptado para su publicación el 7 de agosto de 2020.

Vercelli N; Scaramuzzino RL; D’Alfonso C; Milione G; Piazza G. Cambios en la distribución geográfica de algunas especies americanas en la provincia de Buenos Aires. FAVE - Ciencias Agrarias 20 (1): 33-46 CC BY-NC-SA 4.0 


\begin{abstract}
Changes in the geographical distribution of some American species in the province of Buenos Aires.

Human activities intensification produces severe variations in natural landscapes that could produce changes in species distribution. In Buenos Aires province, plants and animals that extended their habitat to the south were recorded. Here, we analyzed the expansion of Glinus radiatus, Guilleminea densa and Thalia multiflora, which are actually found more than $250 \mathrm{~km}$ far from the southern limit of their known location. Specimens were collected in Azul, Las Flores and Rojas, and then we reviewed the existing bibliography to locate previous records and discuss the causes of their expansion. Among the hypotheses that could explain the progress of these species, regional changes in rainfall and temperature stand out. Its also possible that previously published restricted location is due to insufficient survey, or specimens found could constitute relict populations, or have expanded favored by anthropogenic corridors.
\end{abstract}

Key words: Glinus radiatus, Guilleminea densa, Thalia multiflora, geographical distribution, Buenos Aires province.

\section{INTRODUCCIÓN}

A lo largo del último siglo, los componentes biofísicos de nuestro planeta se han visto alterados como consecuencia de la intensificación de las actividades antrópicas, en un fenómeno conocido como cambio global (OSE, 2011). El crecimiento de la población humana y sus demandas por recursos han generado cambios en el uso del suelo y la energía, provocando aumentos en las concentraciones atmosféricas de gases de invernadero. Así, desde 1880, la temperatura media de la superficie de la Tierra ha aumentado alrededor de $1{ }^{\circ} \mathrm{C}$; en 2018 la temperatura promedio fue $0.83^{\circ} \mathrm{C}$ superior que la media para el periodo 1880-1951, lo que indica una marcada aceleración (NASA-NOAA, 2019).

Numerosos estudios analizaron el efecto del cambio climático sobre la distribución de especies animales y vegetales (McCar- ty, 2001; Walther et al., 2002; Parmesan, 2006; Rosenzweig et al., 2007). Se han documentado diversos efectos en comunidades y ecosistemas debido a la asimetría en el calentamiento que presentan diferentes regiones del mundo, generando heterogeneidad en las dinámicas ecológicas frente a los cambios (Walther, 2010).

En Argentina, particularmente en la provincia de Buenos Aires, durante el siglo XX se ha producido un aumento en los valores medios de precipitación y temperaturas (Berbery et al., 2006; Menéndez, 2006; Barros et al., 2015). Asimismo, se han registrado recientemente numerosas especies animales y vegetales cuya área de distribución se ha expandido hacia el sur de la provincia. Guerrero y Agnolin (2016) documentaron 44 plantas vasculares en el noreste de la provincia típicas de ambientes subtropicales que ampliaron su distribución hacia el sur. Tillansia recurvata (L.) 
L. (Bromeliaceae) ha mostrado un avance de $200 \mathrm{~km}$ hacia el sur con respecto a su distribución conocida (Apodaca y Guerrero, 2019). En el sistema de Tandilia, D’Alfonso et al. (2020) registraron las especies Colletia spinosissima J.F. Gmel. (Rhamnaceae) y Pterocaulon balansae Chodat (Asteraceae), típicas del norte argentino, cuyo límite austral de distribución conocido era el noreste de la provincia de Buenos Aires, así como también Saccharum trinii (Hack.) Renvoize (Poaceae) cuyo límite sur se había determinado en el Delta del Paraná hasta la mitad del siglo XX, hallándose posteriormente poblaciones en la Pampa Deprimida (Vervoorst, 1967).

Glinus radiatus (Ruiz \& Pav.) Rohrb. (Molluginaceae), Guilleminea densa (Humb. \& Bonpl. ex Schult.) Moq. (Amaranthaceae) y Thalia multiflora Horkel ex Körn. (Marantaceae) son especies con localizaciones restringidas en la provincia de Buenos Aires. En los últimos años se han registrado estas especies en áreas de llanura en el interior de la provincia, en algunos casos a más de $250 \mathrm{~km}$ de los extremos sur de distribución consignados hace más de 4 o 5 décadas (Fabris, 1967a; 1967b; 1969; Cabrera y Zardini, 1978). Por lo tanto, el objetivo de este trabajo es dar a conocer las nuevas localizaciones de estas tres especies americanas, compararlas con la distribución registrada previamente, y discutir las causas de su avance hacia el sur.

\section{MATERIALES Y MÉTODOS}

\section{Contexto geográfico}

La extensa planicie del centro-este argentino comprende la región Pampeana, que constituye el ecosistema más importante de praderas del territorio nacional, abarcando la mitad sur de Entre Ríos, el sudeste de Córdoba, sur de Santa Fe, noreste de La Pampa y casi toda la provincia de Buenos Aires. El clima es templado subhúmedo, con precipitaciones entre 700 y 1.200 mm que decrecen de noreste a sudoeste y se concentran en primavera y verano. Las temperaturas medias anuales varían entre 14 y 20 ${ }^{\circ} \mathrm{C}$ y decrecen hacia el sur. La alternancia de periodos secos y húmedos se reconoce en los registros históricos y geológicos; a pesar de esto, desde 1970 se advierte un aumento de las precipitaciones, que han superado los registros previos (Kruse y Laurencena, 2005). En este trabajo, se analizan ejemplares coleccionados en los partidos bonaerenses de Rojas, Azul y Las Flores, perteneciendo el primero a la denominada Pampa Ondulada, y los restantes a la Pampa Deprimida (Figura 1).

La Pampa Ondulada constituye una franja de 120-180 km de ancho que corre paralela al río Paraná desde el Sur de la provincia de Santa Fe, atravesando el Norte de Buenos Aires (hasta Pipinas y Pila) y penetrando en el sudeste de Córdoba (Matteucci, 2012). El paisaje se caracteriza por una suave ondulación del terreno debido a la erosión de los ríos y una leve elevación del basamento cristalino, por lo que se pueden distinguir en esta zona barrancas, bajos y terrazas fluviales. La vegetación natural predominante es el pastizal de Bothriochloa laguroides (DC.) Herter, Nassella neesiana (Trin. \& Rupr.) Barkworth, Pip- 


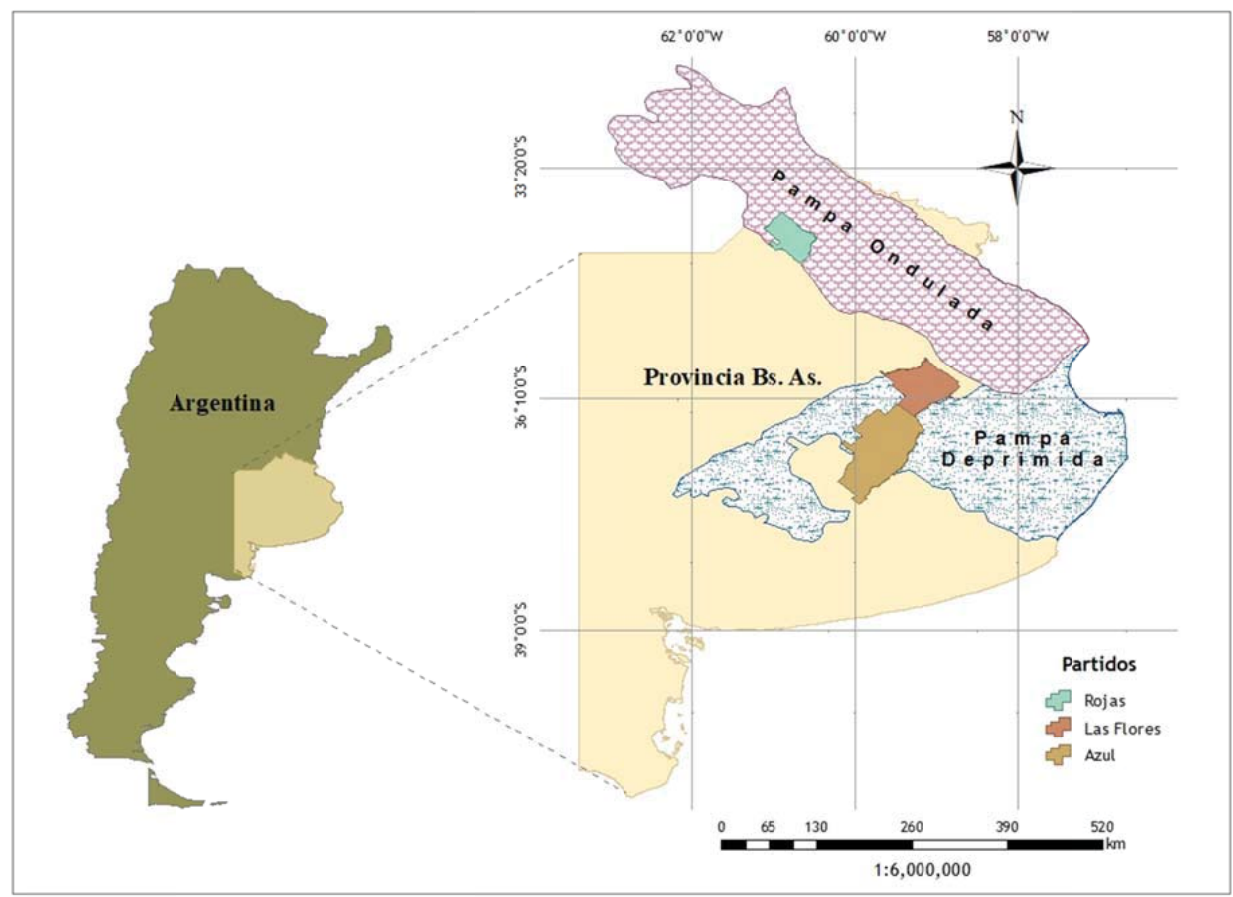

Figura 1. Área de estudio y sitios de colección.

Figure 1. Study area and collection sites

tochaetium montevidensis (Spreng.) Parodi, Aristida murina Cav. y Jarava plumosa (Spreng.) S.W.L. Jacobs \& J. Everett (Matteucci et al., 1999). Sus suelos son los más fértiles y productivos de la región, por lo que la mayor parte de los pastizales se han reemplazado por cultivos y parches de neoecosistemas formados por especies leñosas exóticas, arbustos y hierbas nativas. Por su parte, las barrancas del Paraná y sus tributarios al norte y los cordones de conchilla al este, están cubiertos por bosques xeromórficos de Celtis tala Gillies ex Planch. y Scutia buxifolia Reissek.

La Pampa Deprimida ocupa el centro-este de la provincia de Buenos Aires, formando un triángulo cuya base se encuentra en la costa Atlántica, y se extiende hasta las Sierras de Tandilia. El paisaje de la región presenta un relieve extremadamente llano, con alturas relativas que raramente pasan los 5-10 m y pendientes que varían entre 0,1 y 0,01 \%, y donde dominan geoformas eólicas: mantos irregulares y de baja topografía, y depresiones de deflación. En todo el territorio hay extensas lagunas de aguas estancadas, muchas de ellas sin salida, donde el agua se acumula (Tricart, 1973). En periodos secos, las mencionadas lagunas tienden a disminuir o carecer de agua, conjuntamente con la profundización de la superficie freática. La comunidad natural es el flechillar, donde predominan Chascolytrum subaristatum (Lam.) Desv., Bothriochloa 
laguroides (DC.) Herter, Paspalum dilatatum Poir., Nassella neesiana (Trin. \& Rupr.) Barkworth y Piptochaetium bicolor (Vahl) E. Desv. (Cabrera, 1971), además de pajonales de Paspalum quadrifarium Lam. que se extienden en toda la depresión. Debido a las fuertes limitaciones que presentan los suelos del área por la anegabilidad, alcalinidad, salinidad, muy baja permeabilidad, influencia de la capa freática y escasa profundidad (Hurtado et al., 2005), la actividad principal es la cría extensiva de ganado vacuno.

\section{Taxones estudiados}

Glinus radiatus (Ruiz \& Pav.) Rohrb. es una hierba rastrera con pelos estrellados, hojas pseudoverticiladas, flores en glomérulos y fruto cápsula papirácea. Es una especie tropical o subtropical (Vincent, 2003), que se distribuye desde el sur de Norteamérica hasta Sudamérica austral (Bolivia, Brasil, Chile, Paraguay, Argentina y Uruguay) (Xifreda y Seo, 2006; Zuloaga et al., 2019). En Argentina, habita en lugares húmedos de las Provincias Fitogeográficas Chaqueña, del Espinal y Pampeana, entre los 0 y 500 m.s.n.m. (Xifreda y Seo, 2006) con registros en la provincia de Buenos Aires, CABA, Chaco, Córdoba, Corrientes, Entre Ríos, Formosa y Santa Fe (Zuloaga et al., 2019).

Guilleminea densa (Humb. \& Bonpl. ex Schult.) Moq. es una hierba perenne, rastrera, con pelos lanosos, con hojas opuestas, inflorescencias en glomérulos y fruto utrículo elíptico. Se distribuye desde el sur de Norteamérica hasta el centro de la Argentina, tanto en los Andes como en valles y llanuras (Pedersen, 2000). En nuestro país se encuentra en CABA, Catamarca, Córdoba, Corrientes, Formosa, Jujuy, Salta, Santiago del Estero, Santa Fe y Tucumán (Zuloaga et al., 2019), siendo muy rara cerca de la Cuidad de Buenos Aires (Fabris, 1967a; Cabrera y Zardini, 1978).

Thalia multiflora Horkel ex Körn es una hierba perenne, robusta, con flores violáceas dispuestas en panojas espiciformes y fruto globoso, seco, indehiscente, uniseminado, con pericarpio delgado. Esta especie sudamericana se distribuye en el sur de Brasil, Paraguay, Uruguay y Argentina. En nuestro país habita en las provincias de Chaco, Corrientes, Entre Ríos, Formosa, Santa Fe y noreste de Buenos Aires (Cabrera y Zardini, 1978; Zanotti et al., 2018).

\section{Recolección de datos}

Se colectaron especímenes en diferentes localidades de la provincia de Buenos Aires (Argentina), durante diversos relevamientos florísticos efectuados entre 1990 y 2020. Los ejemplares fueron herborizados, depositados el Herbario de la Facultad de Agronomía de la UNPCPBA (FAA - Thiers, 2020), y determinados con floras regionales y revisiones de géneros (Fabris 1967a; 1967b; 1969; Cabrera y Zardini, 1978; Pedersen, 2000; Vincent, 2003; Xifreda y Seo, 2006; Hurrell y Delucchi, 2008; Zanotti et al., 2018). Adicionalmente se examinaron ejemplares digitalizados en $\mathrm{K}$ y MO correspondientes a las tres especies estudiadas.

Paralelamente se analizaron bases de datos (Flora Argentina, 2020; Portal de Datos Biológicos, 2020; Tropicos, 2020) y diversos trabajos botánicos y fitosociológicos (Burkart, 1957; Vervoorst, 1967; León et al., 1979; Hurrell y Delucchi, 2008), para ubicar registros previos de las especies estudiadas dentro de la provincia de Buenos Aires y luego discutir las causas de su expansión. 


\section{RESULTADOS}

Glinus radiatus (Ruiz \& Pav.) Rohrb.

Recientemente, en el verano de 2020, ha sido hallada en sudoeste de la Pampa Deprimida, en varios sectores de la cuenca baja del Arroyo del Azul (partidos de Azul y Las Flores), tanto en muestreos sistemáticos como ocasionales. Los ejemplares coleccionados habitan en lagunas que se encontraban sin agua en superficie al momento de realizados los muestreos, en algunos casos con alta cobertura de esta especie (Figura 2 A-D).

De acuerdo con la revisión bibliográfica, la distribución geográfica de esta especie en la provincia de Buenos Aires se restringe al Delta del Paraná (en San Nicolás) y alrededores de CABA, específicamente en la zona del puerto (Fabris, 1967b; Cabrera y Zardini, 1978). Hicken (1907; 1910) señaló que sólo la halló en la isla Maciel, en zonas inundadas, y en terrenos de relleno del Río de la Plata, en Puerto Madero, en el año 1911 (Flora Argentina, 2020).

Burkart (1957) la halló en el Delta del Paraná, en la Isla del Pillo (Entre Ríos) asociada a Eragrostis hypnoides (Lam.) Britton, Stern \& Poggenb. (pequeña gramínea rastrera), en suelos desecados a cierta distancia de una laguna; también encontró una comunidad similar en terrenos ganados al Río de la Plata en la década de 1930. Fue citada para el norte de la Pampa Deprimida, en el área Magdalena-Brandsen en relevamientos efectuados en verano, entre 1967 y 1969 (León et al., 1979). A pesar de esto, no fue consignada en los listados florísticos de Vervoorst (1967), quien recorrió la región a fines de la década de 1950 .
En México se la halló en suelos salinos, sitios perturbados y condiciones de sequía en el estado de Guerrero (Vigosa Mercado, 2015), así como en suelos lodosos, orillas de cuerpos de agua y lugares anegados del estado de Aguas Calientes, donde se la considera maleza de cultivos bajo riego por inundación (Sandoval-Ortega y Siqueiros-Delgado, 2018). En Chile, Ramirez et al. (1999) afirman que, en verano, coloniza el centro seco de lagunas primaverales. En países como México y Nicaragua se la considera una especie poco o raramente colectada (Stevens et al., 2001; Vigosa Mercado, 2015).

En el puerto de Buenos Aires, Molfino (1926) mencionó otra especie de este género, Glinus lotoides L., nativa de Eurasia y África (Vincent, 2003), hallada en 1904 y considerada actualmente una cita dudosa para la Argentina (Xifreda y Seo, 2006), dado que el ejemplar de herbario no ha sido hallado. Se ha supuesto que se trató de una presencia accidental (Fabris, 1967b). En Norteamérica estas especies conviven y se han hallado formas intermedias (Vincent, 2003). Los ejemplares coleccionados en la Cuenca del Arroyo Azul corresponden todos a $G$. radiatus.

Material examinado. ARGENTINA. Prov. Buenos Aires: Partido de Azul, Ariel (36 30'0.9”' S - 59 53' 54”' W), 18/01/2020, Milione, Piazza y D'Alfonso s.n. (FAA 10670); Parish, Ea. Santa Elena (36 27' 41'” S - 59 34'36”' O), 20/02/2020, Vercelli, Entraigas, s.n. (FAA 10679). Partido de Las Flores, Pardo, Ea. La Dalinda (36 15’ 32”' S - 59 22’ 13”' O), 19/02/2020, Vercelli, Entraigas, s.n. (FAA 10678). 

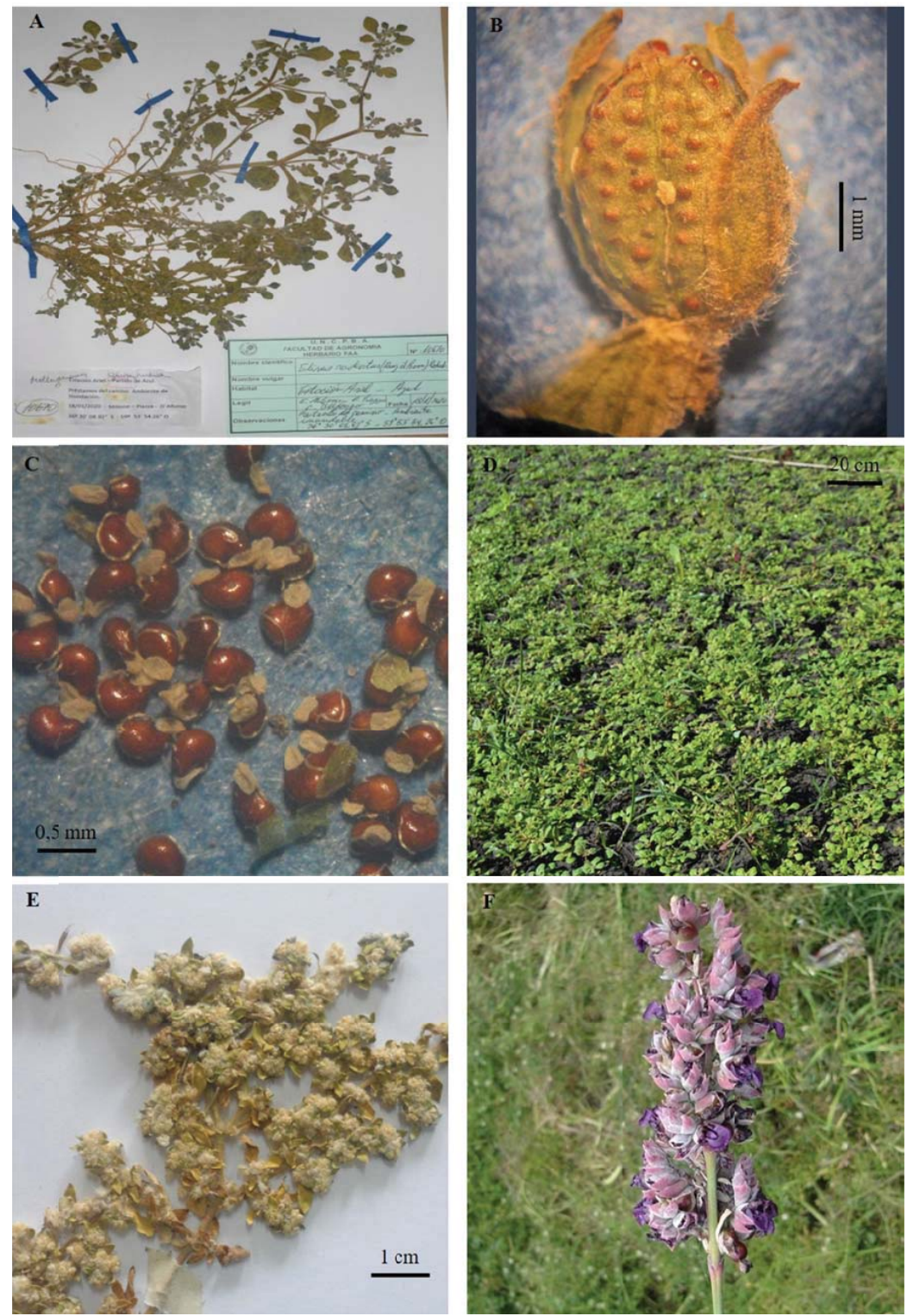

Figura 2. Taxones estudiados. (A-D) Glinus radiatus: (A) Planta (Milione, Piazza y D'Alfonso s.n.; FAA 10670); (B) Fruto; (C) Semillas; (D) Población natural. (E) Guilleminea densa: rama con flores. (F) Thalia multiflora: inflorescencia. Fotos: Carlos D'Alfonso, Genaro Piazza e Ilda Entraigas Figure 2. Analyzed taxa. (A-D) Glinus radiatus: (A) Plant (Milione, Piazza y D'Alfonso w.n.; FAA 10670); (B) Fruit; (C) Seeds; (D) Natural population. (E) Guilleminea densa: branch with flowers. (F) Thalia multiflora: inflorescence. Photography: Carlos D’Alfonso, Genaro Piazza and Ilda Entraigas. 
N. Vercelli et al.

Guilleminea densa (Humb. \& Bonpl. ex Schult.) Moq

Recientemente ha sido hallada en el norte bonaerense (Partido de Rojas), en veredas de la ciudad, en marzo de 2019 (Figura 2 E).

Según la Flora de la provincia de Buenos Aires (Fabris, 1967a), sólo había sido hallada en los adoquines del Puerto de Buenos Aires y en el terraplén del ferrocarril cerca de Campana. Por este motivo, Pedersen (2000) la ha considerado probablemente adventicia en el área y no se ha consignado a la provincia de Buenos Aires en su distribución (Zuloaga et al., 2019). Sin embargo, también ha sido herborizada en el oeste de Buenos Aires, en Trenque Lauquén en 1944 (Flora Argentina, 2020). La revisión bibliográfica indica que esta especie no había sido registrada con anterioridad en la zona ya que, por ejemplo, no fue mencionada por Parodi (1930) en su análisis fitogeográfico del partido de Pergamino.

Material examinado. ARGENTINA. Prov. Buenos Aires: Partido de Rojas, Rojas (30¹1'51”' S - 6044’01”' O), 02/03/2019, Scaramuzzino s.n. (FAA 9395).

Thalia multiflora Horkel ex Körn.

En el interior bonaerense, ha sido hallada en el Arroyo del Azul, con registros desde 1995 hasta el presente, creciendo en forma espontánea en el tramo urbano y suburbano del arroyo (Figura 2 F). Este arroyo, en sus 160 km de extensión aproximada, surca los partidos bonaerenses de Azul y Las Flores (Entraigas, 2013). Nace en el sector serrano de Tandilia para luego adentrarse en la Pampa Deprimida, atravesando primero la ciudad de Azul y, por último, el sector norte del partido homónimo y el sur de Las Flores.

Este arroyo, en sus 160 km de extensión aproximada, surca los partidos bonaerenses de Azul y Las Flores. Nace en el sector se- rrano de Tandilia para luego adentrarse en la Pampa Deprimida, atravesando primera la cuidad de Azul y, por último, el sector norte del partido homónimo y el sur de Las Flores (Entraigas, 2013).

Según la revisión bibliográfica, T. multiflora crece en zonas pantanosas, bañados, embalsados, orillas de cursos de agua, zanjas, cañadas, al costado de vías férreas, en campos de suelos arenosos y al borde de caminos (Zanotti et al., 2018). En la provincia de Buenos Aires, ha sido hallada en el noreste, en zanjas, junto a vías férreas (Fabris, 1969; Cabrera y Zardini, 1978), con registros en Tigre, Pilar y San Isidro. También se ha registrado al sur de CABA, en la ribera platense, hasta la localidad de Atalaya, partido de Magdalena (Hauman, 1917; Hurrell y Delucchi, 2008; Flora Argentina, 2020).

Material examinado. ARGENTINA. Prov. Buenos Aires: Partido de Azul, Arroyo Azul, Camping Municipal (36 48' 00” S 595' 22”' O), 06/12/1995, Orfila, Farina y D'Alfonso s.n. (FAA 2814); Lago Güemes (36 46’ 10”' S - 59 52'14”' O), 03/12/2019, Piazza, D’Alfonso y Scaramuzzino, s.n. (FAA 10945).

\section{DISCUSIÓN}

Los cambios registrados en las precipitaciones, temperaturas y períodos libres de heladas en los últimos años, en especial el desplazamiento $200 \mathrm{~km}$ hacia el sur de la isohieta de $1000 \mathrm{~mm}$, ha sido una de las principales causas planteadas para explicar el avance de muchas especies vegetales y animales subtropicales hacia el sur (Apodaca y Guerrero, 2019; Guerrero y Agnolin, 2016). Esta podría ser también una de las 
razones de la localización de G. radiatus, G. densa y T. multiflora en las llanuras bonaerenses. Sin embargo, pueden señalarse otras hipótesis acerca de la modificación de la distribución geográfica de estas especies.

En primer lugar, se ha considerado que el centro sur bonaerense es un área pobremente muestreada (Guerrero y Agnolin, 2016; D’Alfonso et al., 2020), por lo que la ausencia de registros podría deberse a la insuficiencia de los relevamientos tanto en el espacio como en el tiempo. Especialmente $G$. radiatus y $G$. densa podrían ser especies crípticas por presentar crecimiento postrado y florecer en verano, época donde se realiza pocos muestreos sistemáticos. $G$. densa, además, puede ser confundida con algunas especies de la familia Euphorbiaceae por su porte postrado (Vibrans, 2009), lo que podría dificultar su detección en otras ciudades. Estos casos serían similares al de Colletia spinosissima J. F. Gmel. (D’Alfonso et al., 2020), dado que su presencia en Tandilia no sería debido a un avance de las últimas décadas, sino a la falta de muestreo y sistematización de los registros previos.

Considerando que el área analizada presenta gran variabilidad climática interanual, la hipótesis anterior podría ser muy factible para el caso de $G$. radiatus, ya que ha sido reportada para cuerpos de agua en desecación, situación que no ocurre todos los años. Justamente, en el año 2019 las precipitaciones acumuladas fueron escasas (693 $\mathrm{mm}-$ Estación Azul SMN), en comparación con la media de 916 mm (período 1901-2018), lo que provocó fuertes cambios en el aspecto de las lagunas analizadas (Figura 3)

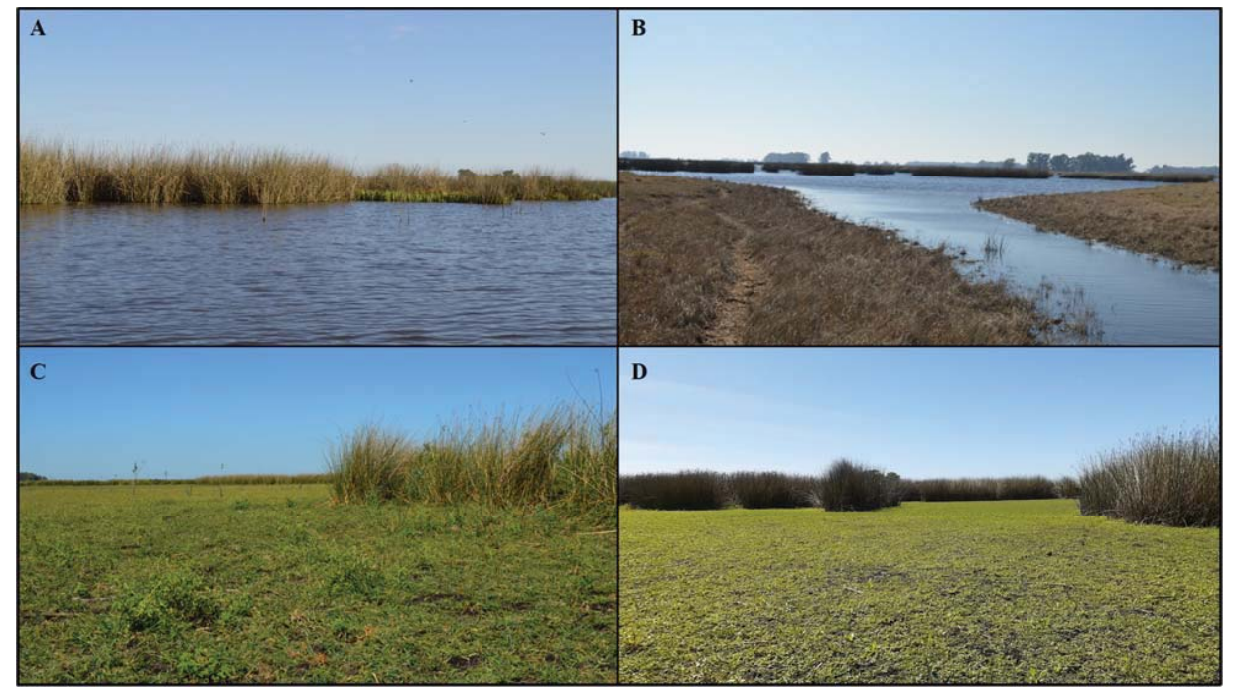

Figura 3. Fisonomía de dos lagunas donde se colecciono Glinus radiatus. Ea. Santa Elena, Parish, Azul, en (A) agosto de 2019 y (C) febrero de 2020 - Ea. La Dalinda, Pardo, Las Flores, en (B) agosto de 2019 y (D) febrero de 2020.

Figure 3. Physiognomy of two lagoons where Glinus radiatus was collected. Ea. Santa Elena, Parish, Azul, in (A) August 2019 and (C) February 2020 - Ea. La Dalinda, Pardo, Las Flores, in (B) August 2019 and (D) February 2020. 
Otras especies del género Glinus, como G. oppositifolius (L.) Aug. DC. y G. lotoides L., presentan propiedades medicinales (Vincent, 2003; Sheu et al., 2014) y han sido reportadas como tóxicas para el ganado (Vincent, 2003; El Ghazali et al., 2008). Aparentemente no existen referencias para $G$. radiatus, pero dada su abundancia en periodos secos, sería conveniente estudiar su toxicidad, así como sus probables usos medicinales. Asimismo, las especies del género Glinus producen semillas con un arilo de origen funicular que finaliza en un estrofíolo (Figura 2 C). Para los casos de G. lotoides y G. opposifolia no se encontró relación entre el arilo y la dispersión por hormigas, ni se halló dormición (Sulakshana y Solomon Raju, 2018), aunque la presencia de estos apéndices en otras especies se ha relacionado con la dispersión zoócora, en particular mirmecocoria (Galán y Castroviejo, 2007). Para el caso de G. radiatus, no se ha estudiado el rol del arilo carnoso de sus semillas y, teniendo en cuenta las escasas colecciones botánicas, es probable que esta especie permanezca en el banco de semillas del suelo largo tiempo, siendo necesario el análisis de otros aspectos de su ciclo de vida que permitan explicar su avance real o aparente durante las últimas décadas, y las razones de su rareza.

Por otro lado, las especies analizadas podrían formar parte de poblaciones relictuales. En el Máximo Térmico del Holoceno (7500-4500 años AP), elementos faunísticos subtropicales llegaron hasta Azul (Tonni, 2017), por lo que podría suponerse que era mayor el número de especies de la flora subtropical en el centro de la provincia de Buenos Aires. Por ejemplo, en la región existen poblaciones relictuales de especies como la orquídea Bipinnula biplumata, que en la provincia de Buenos Aires sólo se distribuye en las sierras de Azul y Olavarría
(Sanguinetti et al., 2015).

Asimismo, T. multiflora y G. densa fueron halladas en las ciudades y sitios próximos, por lo que podrían haberse favorecido por el aumento de temperatura de las ciudades y su funcionamiento como islas de calor. En el caso de G. densa, fue localizada en la cuidad de Rojas entre las baldosas de una vereda, situación similar a su hallazgo entre adoquines en CABA (Fabris, 1967a); su avance hacia el sur se puede haber favorecido por rutas u otros corredores de origen antrópico. A pesar de haber sido registrada en ambientes urbanos, $G$. densa además de ser utilizada como diurética, depurativa de la sangre y para el tratamiento de enfermedades gástricas por campesinos, es considerada maleza en sitios alterados, bordes de caminos y suelos arenosos del Valle de Lerma, Salta (Carrizo y Isasmendi, 1998), así como en otras partes del mundo, por lo que puede haberse trasladado junto con cultivos, maquinarias agrícolas, vehículos y otros. Lo mismo ocurre con T. multiflora, que es considerada maleza de cultivos de arroz en Entre Ríos (Lallana, 2005).

Las poblaciones de T. multiflora en el Arroyo del Azul, es probable que se hayan originado a partir de antiguos cultivos en los espacios verdes públicos que atraviesa el arroyo, ya que es una especie ornamental (Dimitri, 1987). Sin embargo, no existen registros de estos cultivos y desde hace décadas crece en forma espontánea en el tramo urbano y suburbano del arroyo. Si este fuera realmente su origen, debería considerarse que el cultivo de plantas nativas de Argentina en regiones fuera de su área de distribución puede causar alteraciones en los ecosistemas, como en el caso de Limnobium laevigatum (Humb. \& Bonpl. ex Willd.) Heine en el Río Negro, Patagonia (Pérez y Seewald, 2019). 


\section{CONCLUSIONES}

Es probable que el cambio climático sea una de las causas de las modificaciones en los límites de la distribución de las especies analizadas hacia el interior de la provincia de Buenos Aires, dado que son especies tropicales o subtropicales, pero también es necesario considerar otros factores que podrían influir en su expansión. Para los casos de G. radiatus y G. densa una hipótesis muy probable es la ausencia de relevamientos exhaustivos tanto en la Pampa Deprimida como en la gran mayoría de las ciudades del interior bonaerense. Considerando las condiciones ambientales en las que usualmente habita G. radiatus, la hipótesis anterior tiene más relevancia aún dada la frecuencia con las que se presentan años con muy baja pluviometría. Por su parte, la falta de registros históricos en cuanto a las plantas cultivadas en espacios públicos, permite suponer que la expansión de T. multiflora está asociada al factor antrópico, por su condición de especie ornamental.

Se requieren en la provincia de Buenos Aires mayores relevamientos para registrar otras especies del norte argentino que posiblemente habiten en su territorio, y mayor profundidad en la determinación de las causas de su expansión, dado el contexto de cambio global que actualmente predomina. Es probable que especies consideradas de distribución restringida al noreste bonaerense hasta mediados del siglo pasado tengan en el siglo XXI una distribución más amplia.

Dado que el siglo XXI es el siglo de la urbanización, y que se han observado especies nativas raras valiosas de conservar en algunas ciudades europeas, es importante empezar a considerar el rol de las floras urbanas en el contexto de la provincia de Buenos Aires.

\section{AGRADECIMIENTOS}

Se agradece a la Dra. Ilda Entraigas por su participación coleccionando ejemplares y tomando fotografías, a Juan Manuel Goyenetche por su colaboración en la herborización y determinación de ejemplares, y a la Ing. Mg. Luisa Fajardo González por su ayuda en la edición de las figuras. También se agradece a los dueños de las estancias visitadas por permitir el acceso.

\section{BIBLIOGRAFÍA}

Apodaca MJ, Guerrero EL. 2019. ¿Por qué se expande hacia el sur la distribución geográfica de Tillandsia recurvata (Bromeliaceae)? Boletín de la Sociedad Argentina de Botánica 54: 255-261.

Barros VR, Boninsegna JA, Camilloni IA, Chidiak M, Magrín GO, Rusticucci M. 2015. Climate change in Argentina: trends, projections, impacts and adaptation. Wiley Interdisciplinary Reviews: Climate Change 6 (2): 151-169.

Berbery EH, Doyle M, Barros V. 2006. Tendencias regionales en la precipitación. En: Barros V, Clarke R, Silva Días P, editores. El cambio climático en la Cuenca del Plata. Buenos Aires: CONICET. Pp. 67-79.

Burkart A. 1957. Ojeada sinóptica sobre la vegetación del Delta del Río Paraná. Darwiniana 11: 457-560.

Cabrera AL. 1971. Fitogeografía de la República Argentina. Boletín de la Sociedad Argentina de Botánica 14(1-2): 1-42. 
N. Vercelli et al.

Cabrera AL, Zardini E. 1978. Manual de la flora de los alrededores de Buenos Aires. Buenos Aires: Ed. ACME S. A. 755 pp.

Carrizo J, Isasmendi, S. 1998. Amaranthaceae. En: Novara, L, editor. Aportes Botánicos de Salta - Serie Flora 5(4). Salta: Universidad Nacional de Salta. Pp. 1-77.

D’Alfonso CO, Scaramuzzino RL, Gandini ML. 2020. Novedades sobre la distribución de Colletia spinosissima, Pterocaulon balansae y Saccharum trinii en Tandilia (Buenos Aires) y sus posibles causas. Bonplandia, 29 (1): 71-80.

Dimitri MJ. 1987. Marantáceas. En: Dimitri MJ, editor. Enciclopedia Argentina de Agricultura y Jardinería Tomo I (1). Buenos Aires: Ed. Acme. Pp. 280-284.

El Ghazali G, Egami A, Mohammed M, Abdalla WE, Mohammed H. 2008. Poisonous Plants of the Sudan. Khartoum, Sudan: National Centre For Research, Ministry of Science and Technology. https://www.researchgate. net/publication/301892397_Poisonous_ Plants_of_Sudan

Entraigas I. 2013. El arroyo del Azul. En: Entraigas I, Vercelli N, editores. Los paisajes de la cuenca del arroyo del Azul. Editorial Martín, Mar del Plata, pp. 29-36.

Fabris H. 1967a. Amaranthaceae. En: Cabrera AL, editor. Flora de la Provincia de Buenos Aires. Tomo IV. Buenos Aires: Colección Científica INTA. Pp. 127-192.

Fabris H. 1967b. Aizoaceae. En: Cabrera AL, editores. Flora de la Provincia de Buenos Aires. Tomo 4 (3). Buenos Aires: Colección Científica INTA. Pp. 171-180.

Fabris H. 1969. Marantaceae. En: Cabrera AL, editores. Flora de la Provincia de Buenos Aires. Tomo 4 (1). Buenos Aires: Colección Científica INTA. Pp. 571-574.

Flora Argentina. 2020. (Consulta 2020 febrero 28). http://www.floraargentina.edu.ar
Galan A, Castroviejo S. 2007. Araceae. En: Castroviejo S, editores. Flora Ibérica XVIII. Madrid: Real Jardín Botánico. Pp. 282-310.

Guerrero EL, Agnolin FL. 2016. Recent changes in plant and animal distribution in the southern extreme of the Paranaense biogeographical province (northeastern Buenos Aires province, Argentina): Ecological responses to climate change? Revista del Museo Argentino de Ciencias Naturales nueva serie 18: 9-30.

Hauman L. 1917. Notes floristiques. An. Mus. Nac. Hist. Nat. Buenos Aires 29: 391-443.

Hicken C. 1907. Notas Botánicas. Anales de la Sociedad Científica Argentina 65: 290-312.

Hicken C. 1910. Chloris platensis argentina. Ap. Hist. Nat 2: 1-292.

Hurrell J, Delucchi G. 2008. Marantaceae. En: Hurrell J, editores. Flora Rioplatense. Parte 3: Monocotiledóneas. Vol. 1 (Alismatales, Arecales, Commelinales, Zingiberales). Buenos Aires: Editorial L.O.L.A. Pp. 163-174.

Hurtado MA, Moscatelli GN, Godagnone RE. 2005. Los suelos de la provincia de Buenos Aires. En: Barrio RE, Etcheverry RO, Caballé MF, Llambías E, editores. Geología y Recursos Minerales de la Provincia de Buenos Aires. La Plata: Relatorio del XVI Congreso Geológico Argentino. Pp. 201-218.

Kruse E, Laurencena P. 2005. Aguas superficiales. Relación con el régimen subterráneo y fenómenos de anegamiento. En: Barrio RE, Etcheverry RO, Caballé MF, Llambías E, editores. Geología y Recursos Minerales de la Provincia de Buenos Aires. La Plata: Relatorio del XVI Congreso Geológico Argentino. Pp. 313-326.

Lallana VH. 2005. Lista de malezas del cultivo de arroz en Entre Ríos, Argentina. Revista Ecosistemas 14 (2): 162-167. 
León RJC, Burkart SE, Movia CP. 1979. Relevamiento Fitosociológico del Pastizal del Norte de la Depresión del Salado: La Vegetación de la República Argentina. Serie Fitogeográfica $\mathrm{N}^{\circ}$ 17. Buenos Aires: INTA. 90 p.

Matteucci SD. 2012. Ecorregión Pampa. En: Morello J, Matteucci SD, Rodríguez AF, Silva $\mathrm{M}$, editores. Ecorregiones y Complejos Ecosistémicos Argentinos. $1^{\circ}$ Edición. Buenos Aires: Orientación Gráfica Editora. Pp. 391-446.

Matteucci, SD, Morello J, Rodríguez A, Buzai GD, Baxendale C. 1999. El crecimiento de la metrópoli y los cambios de biodiversidad: el caso de Buenos Aires. En: Matteucci SD, Solbrig OT, Morello J, Halffter G, editores. Biodiversidad y uso de la tierra. Conceptos y ejemplos de Latinoamérica. Buenos Aires: EUDEBA-UNESCO. Pp. 549-580.

McCarty JP. 2001. Ecological consequences of recent climate change. Conserv. Biol. 15 (2): 320-331.

Menéndez A. 2006. Tendencias hidrológicas en la Cuenca del Plata. En: Barros V, Clarke R, Silva Días P, editores. El cambio climático en la Cuenca del Plata. Buenos Aires: CONICET. pp. 81-92.

Molfino JF. 1926. Adiciones a la flora fanerogámica adventicia de la Argentina. Anal. Mus. Hist. Nat. Buenos Aires. 34: 89-119.

NASA-NOAA. 2019. 2018 Fourth Warmest Year in Continued Warming Trend. NASA's Goddard Institute for Space Studies - National Oceanic and Atmospheric Administration, USA. (Consulta 2020 febrero 28). https://www.nasa.gov/press-release/2018fourth-warmest-year-in-continued-warmingtrend-according-to-nasa-noaa

OSE. 2011. Biodiversidad en España. Bases de la sostenibilidad ante el cambio global. Observatorio de la Sostenibilidad en España, Madrid. 503 p.
Parmesan C. 2006. Ecological and evolutionary responses to recent climate change. Annual Review of Ecology, Evolution, and Systematics 37: 637-669.

Parodi L. 1930. Ensayo fitogeográfico sobre el Partido de Pergamino. Revista de la Facultad de Agronomía y Veterinaria de Buenos Aires 7: 65-271.

Pedersen TM. 2000. Studies in South American Amaranthaceae V. Bonplandia 10 (1-4): 83112.

Pérez CH, Seewald P. 2019. Limnobium laevigatum (Humb. \& Bonpl. Ex Willd.) Heine (Alismatales, Hydrocharitaceae), una nueva especie invasora en la cuenca del Río Negro, Provincia De Río Negro, Argentina. Revista Historia Natural 9 (2): 211-218.

Portal de Datos de Biodiversidad Argentina. 2020. (Consulta 2020 febrero 28). https://datos.sndb.mincyt.gob.ar/

Ramírez C, San Martín C, Ojeda P. 1999. Estudio de la flora de comunidades ruderales antropogénicas en la IX Región de la Araucanía, Chile. Studia Botánica, 18: 47-68.

Rosenzweig C, Casassa G, Karoly DJ, Imeson A, Liu C, Menzel A, Rawlins S, Root TL, Seguin B, Tryjanowski P, Hanson CE. 2007. Assessment of observed changes and responses in natural and managed systems. En: Parry ML, Canziani OF, Palutikof JP, van der Linden PJ, editores. Climate Change 2007: Impacts, Adaptation and Vulnerability. Contribution of Working Group II to the Fourth Assessment Report of the Intergovernmental Panel on Climate Change. Reino Unido: Cambridge University Press. Pp. 79-131.

Sandoval-Ortega MH, Siqueiros-Delgado ME. 2018. Las Familias Aizoaceae, Molluginaceae y Phytolaccaceae (Caryophyllales) en el Estado de Aguascalientes, México. Polibotánica, 46: 27-47. 
N. Vercelli et al.

Sanguinetti A, Buzatto CR, Singer RB. 2015. Vervoorst FB. 1967. La vegetación de la ReThe genus Bipinnula (Orchidaceae: Chloraeinae) in Argentina. Nordic Journal of Botany. 33(4): 421-431

Sheu SY, Yao CH, Lei YC, Kuo TF. 2014. Recent progress in Glinus oppositifolius research. Pharmaceutical biology, 52(8): 10791084

Stevens WD, Ulloa Ulloa C, Pool A, Montiel Jarquín OM. 2001. Flora de Nicaragua. Monogr. Syst. Bot. Missouri Bot. Gard. 85: ixlii.

Sulakshana M, Solomon Raju AJ. 2018. Floral biology and pollination of carpet weeds, Glinus lotoides L. and Glinus oppositifolius (L.) Aug. DC. (Molluginaceae). Anales de Biología 40: 103-114.

pública Argentina. VII. Las comunidades vegetales de la depresión del Salado. Serie Fitogeográfica $\mathrm{N}^{\circ} 7$. Buenos Aires: INTA. 262 pp.

Vibrans H. 2009. Malezas de México, CONABIO. (Consulta 2020 marzo 23). http://www. conabio.gob.mx/malezasdemexico/amaranthaceae/guilleminea-densa/fichas/ficha.htm

Vigosa Mercado JL. 2015. Molluginaceae. En: Jiménez J, Fonseca RM, Martínez M, editores. Flora de Guerrero. México: Prensas de Ciencias. Pp. 13-26.

Vincent M. 2003. Molluginaceae. En: Flora of North America Editorial Committee (Eds.). Fl. N. Amer. 4. New York: Oxford University Press. Pp. 509-511.

Thiers B. 2020. Index Herbariorum: a global Walther GR. 2010. Community and ecosystem directory of public herbaria and associated staff. New York Botanical Garden's Virtual Herbarium. (Consulta 2020 marzo 27). http:// sweetgum.nybg.org/ih

Tonni EP. 2017. Cambios climáticos en la región pampeana oriental durante los últimos 1000 años. Una síntesis con énfasis en la información zoogeográfica. Revista del Museo de La Plata 2: 1-11.

Tricart JL. 1973. Geomorfología de la Pampa Deprimida. Base para los estudios edafológicos y agronómicos. INTA XII, Colección Científica. 202 pp.

Trópicos. 2020. Missouri Botanical Garden. (Consulta 2020 febrero 28). http://www.tropicos.org. responses to climatic change. Philosophical Transactions of the Royal Society of London, B: Biological Sciences 365: 2019-2024.

Walther GR, Post E, Convey P, Menzel A, Parmesan C, Beebee T, Fromentin J, Hoegh-Guldberg O, Bairlein F. 2002. Ecological responses to recent climate change. Nature 416: 389-395.

Xifreda CC, Seo MN. 2006. Molluginaceae. En: Anton AM, Zuloaga FO, editores. Flora Fanerogámica Argentina 100: 1-5

Zanotti CA, Ospina JC, Keller HA. 2018. Sinopsis y novedades taxonómicas de la familia Marantaceae en la Argentina, incluyendo una nueva cita para el Paraguay. Boletín de la Sociedad Argentina de Botánica 53(3): 1-10. Zuloaga FO, Belgrano MJ, Zanotti CA. 2019. Actualización del Catálogo de las Plantas Vasculares del Cono Sur. Darwiniana, nueva serie, 7(2): 208-278 (con apéndices). 